Check for updates

Lancashire County Council

Cite this as: BMJ 2022;376:0185 http://dx.doi.org/10.1136/bmj.0185 Published: 21 January 2022

\section{Omicron and the NHS: we need to look beyond hospital care to solve the pressures}

\author{
Sakthi Karunanithi Director of Public Health
}

Even though Plan B measures in England were activated in December, along with a booster programme of vaccination, cases of the omicron variant of SARS-CoV-2 have continued to rise in the six weeks since it was first detected in the UK, particularly among those aged 60 and above. It is only in the past couple of weeks that cases have started to fall, initially in London.

Although use of intensive care units and deaths have not been as high relative to case rates as with other variants of covid-19, the arrival of omicron variant has led to several NHS hospital trusts declaring internal critical incidents. ${ }^{1}$ This is due to the twin problems of rising levels of staff sickness due to covid-19 along with an increasing number of hospital admissions in general and acute beds.

NHS trusts declaring internal critical incidents is not uncommon during the winter months and frequently signals the prioritisation of urgent and emergency services over elective procedures within the Trusts. NHS trusts are experiencing long delays in ambulance handovers, busy emergency departments, and omicron associated nosocomial infections, which are limiting visitors to hospitals. The already long backlogs of elective procedures are further delayed and there are delays in discharging people out of hospital and back into their own homes or to social care settings. With urgent and critical services prioritised, staff are being redeployed internally to deal with the immediate issues. Furthermore, the NHS has been setting up new Nightingale surge hubs at hospitals across the country. ${ }^{2}$

Without a concurrent increase in the staffing, particularly in social care capacity in the community, these surge beds are likely to be no more than additional holding beds with staff shortages leading to patients experiencing delayed discharges to their own homes or to social care.

As well as the direct impact on hospitals and patients, declaring a critical incident has additional opportunity costs and indirect health impacts. The root causes of these critical incidents can be traced back to three fundamental problems:

1. We count what can be measured and don't measure what really counts

While the national covid dashboards report on the hospital situation daily, rarely do similar statistics get published to describe the state of primary care, social care, community, and mental health services, who are also dealing with the same problems, and could become essential part of the solution, if this was planned more effectively.
2. We have constrained the main solutions for hospital-based problems to be within the walls of hospitals.

Rather than only focusing on hospital care, we should expand our focus to supporting people better in the community, including through voluntary, community, and faith sectors. For example, the implementation of covid oximetry at home and virtual wards can help to detect people at risk of deterioration early and support stable patients better at home. Investing adequately in a sustainable social care system could negate the need for nightingale surge hubs. Yet, we have somehow turned a blind eye to measuring and supporting primary care and social care as solutions to fortify the NHS.

3. We often ignore signals of workload distress from the frontline.

Despite NHS and social care being a people driven endeavour, we have overlooked the need for sustainable workforce planning across health and social care at our peril. ${ }^{34}$ As the saying goes, we should not let this crisis go to waste. If we are to prepare seriously to respond to future waves of covid-19 and prepare for a strong recovery, we need to start listening to the critical intelligence from the front line and commit to delivering a sustainable workforce plan as a matter of urgency.

Even though internal critical incidents have been declared over recent weeks by a number of NHS hospital trusts, they also represent deeper and often overlooked areas for investment and action beyond the boundaries of our hospitals. To be able to hold these apparently opposing ideas and yet be able to find sustainable solutions, should become the hallmark of sustaining health and care systems through the crises of today and the future.

Competing interests: none declared

Provenance and peer review: commissioned, not peer reviewed

lacobucci G. Covid-19: NHS trusts declare "critical incidents" because of staff shortages. BM/2022;376:03. doi: 10.1136/bmj.03. pmid: 34983792

2 NHS plans new Nightingale facilities in response to Omicron. December 2021. https://www.england.nhs.uk/2021/12/nhs-plans-new-nightingale-facilities-in-response-to-omicron/

3 Parliament UK. Omicron and emergency care crisis could derail plans to tackle backlog, warn MPs. January 2022. https://committees. parliament.uk/committee/81/health-and-social-care-committee/news/160095/omicron-and-emergency-care-crisis-could-derail-plans-to-tackle-backlog-warnmps/

Parliament UK. Workforce planning. https://publications.parliament.uk/pa/cm5802/cmselect/cmhealth/22/2208.htm 